\title{
Effect of sumac extract on serum oxidative status, RANKL/OPG system and alveolar bone loss in experimental periodontitis in rats
}

\author{
1- Department of Periodontology, School of Dentistry, Izmir Katip Celebi University, Izmir, Turkey. \\ 2- Department of Periodontology, School of Dentistry, Akdeniz University, Antalya, Turkey. \\ 3- Department of Pathology, School of Medicine, Necmettin Erbakan University, Konya, Turkey. \\ 4- Department of Chemistry, Faculty of Sciences and Arts, Erzincan University, Erzincan, Turkey.
}

Mehmet SAĞLAM¹, Serhat KÖSEOĞLU', Mükerrem HATiPOĞLU², Hacı Hasan ESEN³ , Ekrem KÖKSAL ${ }^{4}$

Corresponding address: Mehmet Sağlam - Izmir Katip Celebi University, Faculty of Dentistry, Department of Periodontology - Izmir - Turkey - GSM: +90 (0530) 3249948 - e-mail: dtmehmetsaglam@hotmail.com

Submitted: August 7, 2014 - Modification: October 12, 2014 - Accepted: October 14, 2014

\section{ABSTRACT}

$\mathrm{O}$ bjectives: Sumac (Rhus coriaria L.) is widely used spice which has several properties such as antioxidant, anti-inflammatory and antimicrobial. The purpose of this animal study was to evaluate the effects of sumac extract on levels of receptor activator of nuclear factor-kappa B ligand (RANKL), osteoprotegerin (OPG) expression, serum oxidative status, and alveolar bone loss in experimental periodontitis. Material and Methods: Twenty-four Wistar rats were separated into three groups: non-ligated $(\mathrm{NL}, \mathrm{n}=8)$, ligature only ( $L O$, $\mathrm{n}=8)$, and ligature and treated with sumac extract $(S, n=8)(20 \mathrm{mg} / \mathrm{kg}$ per day for 11 days). A 4/0 silk suture was placed around the mandibular right first molars subgingivally; after 11 days, the rats were sacrificed, and alveolar bone loss was histometrically measured. The detection of RANKL and OPG were immunohistochemically performed. Levels of serum total antioxidant status (TAS)/total oxidant status (TOS), and oxidative stress index (OSI) were also analyzed. Results: Alveolar bone loss was significantly greater in the LO group compared to the $S$ and NL groups $(p<0.05)$. The number of inflammatory cell infiltrate (ICI) and osteoclasts in the LO group was significantly higher than that of the NL and S groups $(p<0.05)$. The number of osteoblasts in the $L O$ and $S$ groups was significantly higher than that of the NL group $(p<0.05)$. There were significantly more RANKL-positive cells in the LO group than in the $S$ and NL groups $(p<0.05)$. OPG-positive cells were higher in $S$ group than in LO and NL groups $(p<0.05)$. TOS and OSI levels were significantly reduced in $\mathrm{S}$ group compared to LO group $(\mathrm{P}<0.05)$ and TAS levels were similar in $\mathrm{S}$ and $\mathrm{NL}$ group $(p>0.05)$. Conclusions: The present study showed that systemic administration of sumac extract may reduce alveolar bone loss by affecting RANKL/OPG balance, TOS and OSI levels in periodontal disease in rats.

Keywords: Rhus. Alveolar bone loss. Periodontal diseases. Receptor activator of nuclear factor-kappa B ligand. Osteoprotegerin.

\section{INTRODUCTION}

Sumac is widely used as a spice, also as a herbal cure in traditional medicine because of its anti-inflammatory, antimicrobial, and antioxidant properties ${ }^{26}$. Periodontitis is a chronic inflammatory disease triggered by pathogenic microorganisms and the progression of destructive disease seems to be associated with an abnormal host response to those microorganisms ${ }^{25}$. Host cells (e.g., polymorphonuclear leukocytes) release reactive oxygen species (ROS) as a part of the immune response against to microbial attacks ${ }^{28}$. Excessive production of ROS by polymorphonuclear leukocytes plays an important role in the periodontitis pathogenesis, and it causes periodontal tissue damage by oxidizing DNA, proteins, and lipids ${ }^{13}$. It was indicated that ROS serve as an intracellular signal mediator for osteoclast differentiation ${ }^{21}$. It was reported that ROS are capable of inducing 
periodontal tissue destruction and are associated with osteoclastic bone resorption ${ }^{30}$.

RANKL is a membrane-bound protein that belongs to the tumor necrosis factor (TNF) ligand superfamily. It is responsible for stimulation of osteoclast differentiation and bone resorption?. Osteoprotegerin (OPG) is a soluble tumor necrosis factor receptor-like molecule that serves as a decoy receptor and blocks the binding of RANKL to RANK and thus prevents osteoclastogenesis. The balance between RANKL and OPG levels regulates the bone tissue destruction occurred in periodontitis ${ }^{6}$. It was suggested that sites with active bone resorption present a differential pattern of RANKL/OPG expression when compared to sites where bone resorption is absent or minimal ${ }^{22}$. It was indicated that sites where expression of RANKL predominates (RANKL>OPG) are putative progressive lesions, whereas sites where expression of RANKL and OPG

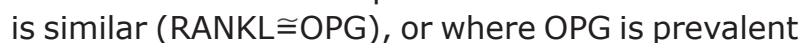
(RANKL<OPG), are potentially stable lesions ${ }^{22}$.

Sumac (Rhus coriaria L.) is a well-known spice belonging to Anacardiaceae family and grows in a wide region such as Mediterranean countries, South Europe, North Africa, Iran, and Afghanistan ${ }^{24}$. In the literature, the antioxidant, antimicrobial, and anti-inflammatory properties of alcoholic (methanol/ethanol) and water extracts of sumac is well documented. It was reported that methanolic Sumac fruits extracts have marked antioxidant activity against lipid peroxidation and free radicals in vitro ${ }^{9}$. Water extracts of sumac have remarkable antioxidant and radical scavenging properties ${ }^{8}$. In other studies, it has been demonstrated that gallic acid, the active principle of sumac, is a crucial-antioxidant which defends against ROS-induced DNA-damage ${ }^{11,16}$. Considering the anti-inflammatory properties of sumac, it has been reported that lyophilized hydroalcoholic crude extract of sumac reduced inflammation, chondrodegeneration, and oxidative stress in osteoarthritis ${ }^{29}$. It was also reported that tannic acid, which is present in sumac extract, is effective to inhibit the proteolytic activities of the periodontopathic bacteria Porphyromonas gingivalis, Bacteroides intermedius, and Treponema denticola ${ }^{18}$.

Based on these favorable aspects of sumac extract, we hypothesized that it is a beneficial antioxidant agent in suppressing periodontal inflammation and alveolar bone destruction in periodontal disease. Therefore, the present study investigates the effects of systemic sumac extract administration on alveolar bone loss by evaluating the changes in RANKL/OPG balance and serum oxidative status in experimental rat periodontitis.

\section{MATERIAL AND METHODS}

\section{Experimental design}

All experimental procedures were approved by the Animal Ethics Committee of Selcuk University School of Medicine (No: 2012-013) for the use on animal experiments. Twenty-four male Wistar rats (weight: $340 \pm 10 \mathrm{~g}$ ) were separated into three groups: non-ligated (NL), ligature only (LO), and ligature and treated with sumac (S) $(20 \mathrm{mg} / \mathrm{kg}$ per day for 11 days). Eight rats were randomly assigned to $\mathrm{NL}, \mathrm{LO}$, and $\mathrm{S}$ groups. They were housed in wire cages and maintained on a $12 \mathrm{~h}-12 \mathrm{~h}$ light-dark cycle with a constant room temperature of $25^{\circ} \mathrm{C}$.

\section{Preparation of sumac extract}

The fruits of sumac were collected from a spice store from Erzincan, Turkey. The sumac fruits were prepared as powder form and stored at $25^{\circ} \mathrm{C}$ until use. Sumac extract was prepared by an experienced chemist (EK) in accordance with previously published method ${ }^{8}$. Twenty-five gram sample of sumac was added to $250 \mathrm{ml}$ ethanol and this admixture was stirred using a magnetic stirrer during $10 \mathrm{~h}$ in the preparation of ethanol extraction. The filtrates were evaporated after the extract was filtered by using filter paper. Thus, in that way ethanol extract of sumac was produced. Prepared sumac extract was stored at $-30^{\circ} \mathrm{C}$ until use.

\section{Administration of sumac extract}

Experimental periodontitis in rats was performed as in our previous study ${ }^{27}$. Animals were anesthetized with ketamine (Eczacıbasi Ilac Sanayi, Istanbul, Turkey) $(40 \mathrm{mg} / \mathrm{kg}$ ) and the same operator $(\mathrm{MH})$ placed a 4/0 silk suture (Sterisilk, SSM Sterile Health Products Inc, Istanbul, Turkey) around the right first molars in mandible subgingivally to stimulate experimental periodontitis. The animals were housed in individual wire cages and received water and food ad libitum.

In the test group, the rats received sumac orogastrically, at a dosage of $20 \mathrm{mg} / \mathrm{kg} /$ day at placement of the ligature and daily until sacrifice. The ligatures were kept subgingivally for 11 days. After 11 days, blood samples were obtained, the rats were sacrificed and their mandibles were split in two equal pieces from the midline between the incisor teeth after surgical dissection. The right pieces of the mandibles were sent to a pathologist laboratory for histologic examination. To obtain serum, the blood samples were centrifuged at 3000x G for $10 \mathrm{~min}$. Subsequently, the serum samples were collected into a sterile polypropylene tube and kept at $-80^{\circ} \mathrm{C}$ until being analyzed for the total antioxidant/oxidant status and oxidative stress index (TAS, TOS, OSI). 


\section{Histometric analysis}

The samples were fixed in $10 \%$ formalin for 72 hours. They were decalcified in 10\% EDTA for 60 days and embedded in paraffin. Mesio-distally serial sections $(5 \mu \mathrm{m})$ were taken and stained by using hematoxylin and eosin solutions. For histometric evaluation, three equally distant sections belonging to each molar were chosen. The distance between the cemento-enamel junction (CEJ) and the alveolar bone crest $(A C)$ was measured to determine histometric-alveolar bone loss. Evaluation was carried out by a single examiner (HHE) blinded to the treatment groups using an image analysis system (BAB Bs200ProP Image Analysis System, BAB Mühendislik MÜ, Ankara, Turkey) connected to a light microscope (Olympus BX51, Tokyo, Japan) (40x magnification). For assessing the bone level, three linear measurements were obtained on the mesial and distal sides (six linear measurements at total). A mean value was calculated for each tooth.

\section{Histopathologic evaluation}

The connective tissue areas under the epithelium and interdental septum were evaluated under light microscopy. Two sections $(5 \mu \mathrm{m})$ at $200 x$ magnification were used for light microscopy assessment. The number of osteoblasts, osteoclasts, and inflammatory cell infiltrate (ICI) were analyzed in connective tissue and interdental septum. All of the osteoblasts (mononuclei, cuboidal cells around the active bone formation surfaces) and osteoclasts (multiple nuclei, ruffled border, granular cytoplasm) were counted in the periodontium morphologically. TRAP staining was performed to identify and quantify osteoclasts (Sigma Chemical Co., St Louis, MO, USA).

\section{Immunohistochemistry}

For the immunostaining procedure, anti-rabbit RANKL polyclonal antibody and anti-rabbit OPG polyclonal antibody (Abcam, Cambridge, MA, USA) were used. Immunohistochemical analyses were performed as described in our previous study ${ }^{27}$. Morphometric analysis was performed in connective tissue under the epithelial basement membrane and interdental septum for the quantitative evaluation of immunolabeled cells. The detection of the number of immunolabeled cells per unit area (number of cells $/ \mathrm{mm}^{2}$ ) for each reagent was performed with a light microscope at 400x magnification. A camera (Panasonic, Osaka, Japan) equipped microscope was used and each microscopic field was analyzed by using the same image analysis system. Three randomly microscopic regions were selected per stained section for immunolabeled cell counting, and the data for each rat represent the mean of 3 quantifications. All measurements were performed by the same masked examiner (HHE).
TAS, TOS, and OSI levels of serum samples

\section{Total antioxidant status (TAS)}

Serum TAS was analyzed by using a commercially available kit [Fully Automated $3^{\text {rd }}$ Generation Total Antioxidant Status (TAS) ASSAY KIT Product Code: RL0017, REL Assay Diagnostics, Mega TIp, Gaziantep, Turkey] developed by Erel ${ }^{14}$ (2004). In this assay, the antioxidative effect of the sample against a potent free-radical reaction initiated by the hydroxyl radical produced is measured. The results are obtained as millimoles of trolox equivalent per liter ( $\mathrm{mmol}$ trolox equiv/l).

\section{Total oxidant status (TOS)}

Serum TOS was analyzed by using a commercially available kit [Fully Automated Total Oxidant Status (TOS) ASSAY KIT Product Code: RL0024, REL Assay Diagnostics, Mega Tıp, Gaziantep, Turkey] developed by Erel $^{14}$ (2004). The assay is calibrated with hydrogen peroxide, and the results are obtained in micromolar hydrogen peroxide equivalent per liter ( $\mu \mathrm{mol} \mathrm{H}_{2} \mathrm{O}_{2}$ equiv/l).

\section{Oxidative stress index (OSI)}

OSI is an indicator of the degree of oxidative stress. The percentage ratio of the TOS to the TAS is used to calculate OSI. To perform the calculation, the result unit of TAS, millimole of Trolox equivalent per liter, was converted to micromole equivalent per liter, and the OSI value was calculated by the formula OSI $=[($ TOS,$\mu \mathrm{mol} / \mathrm{L}) /($ TAS,$\mu \mathrm{mol}$ Trolox equivalent/L) $\times 100]$.

\section{Statistical analysis}

The statistical analysis was performed using a commercially available software program (SPSS 20.0, IBM, Chicago, IL, USA). The Shapiro-Wilk's normality test was used to verify the normality of the data. The data were normally distributed and parametric tests were used. ANOVA test followed by Tukey's test for pair-wise comparisons were used for analyzing the number of osteoclast, osteoblast, inflammatory cells, RANKL and OPG positive cells, alveolar bone loss, TAS, TOS and OSI values. A value of $p<0.05$ was considered statistically significant.

\section{RESULTS}

\section{Histometric findings}

Significantly greater alveolar bone loss was observed in the LO group compared to $\mathrm{S}$ and NL groups $(P<0.05$; Figure $1 \mathrm{~A})$. The alveolar bone loss was significantly greater in the $\mathrm{S}$ group than in the NL group $(P<0.05)$.

\section{Histopathologic findings}

The inflammatory infiltrate $\left(\mathrm{mm}^{2}\right)$ numbers in the study groups were presented in Figure 1B. The number of inflammatory infiltrate was greater in 
the LO group compared with the $\mathrm{S}$ and NL groups $(p<0.05)$. There was also significant difference between $S$ and $N L$ groups $(p<0.05)$.

Figure $1 \mathrm{C}$ presents number of osteoclasts $\left(\mathrm{mm}^{2}\right)$ in the study groups. Except for one in the NL group, osteoclasts were observed in all rats. Significantly higher numbers of osteoclasts were observed in the LO group compared with the other two groups $(p<0.05)$. Significant difference was also found between $S$ and NL groups $(p<0.05)$.

The number of osteoblasts $\left(\mathrm{mm}^{2}\right)$ was presented in Figure 1D for the study groups. Osteoblasts were found in all rats. The osteoblasts numbers were greater in the LO and S groups compared with NL group $(p<0.05)$. The osteoblast numbers were similar in LO and S groups ( $p>0.05)$.

The histological images representative of each experimental group are shown in Figure 2.

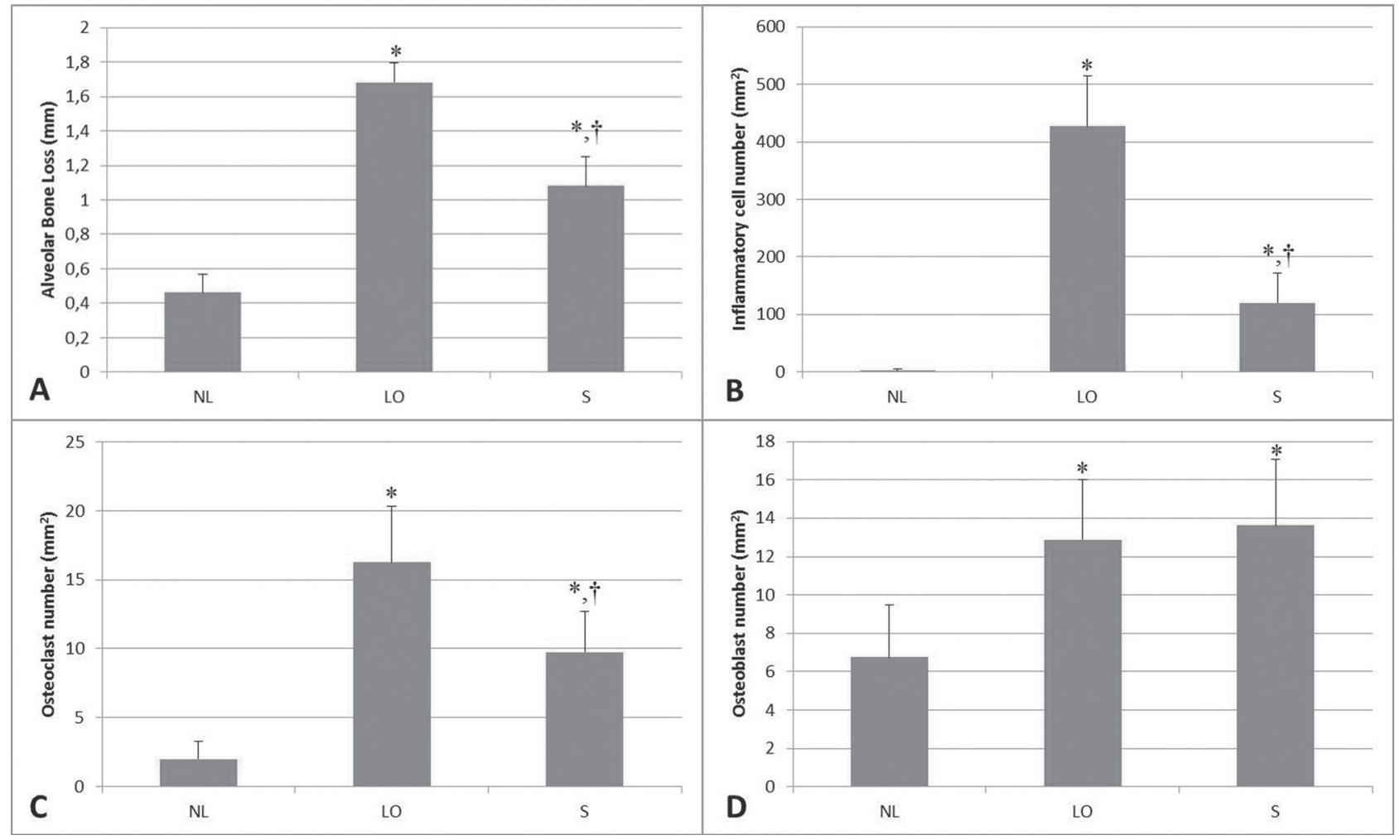

$\mathrm{NL}=$ non-ligated; $\mathrm{LO}=$ ligature only; $\mathrm{S}=$ ligature and treated with sumac

Figure 1- A- Mean alveolar bone loss in groups; B- The number of inflammatory infiltrate $\left(\mathrm{mm}^{2}\right)$ in the study groups; C- The number of osteoclasts $\left(\mathrm{mm}^{2}\right)$ in the study groups; D- The number of osteoblasts $\left(\mathrm{mm}^{2}\right)$ in the study groups. *Significant difference compared to NL group. †Significant difference compared to LO

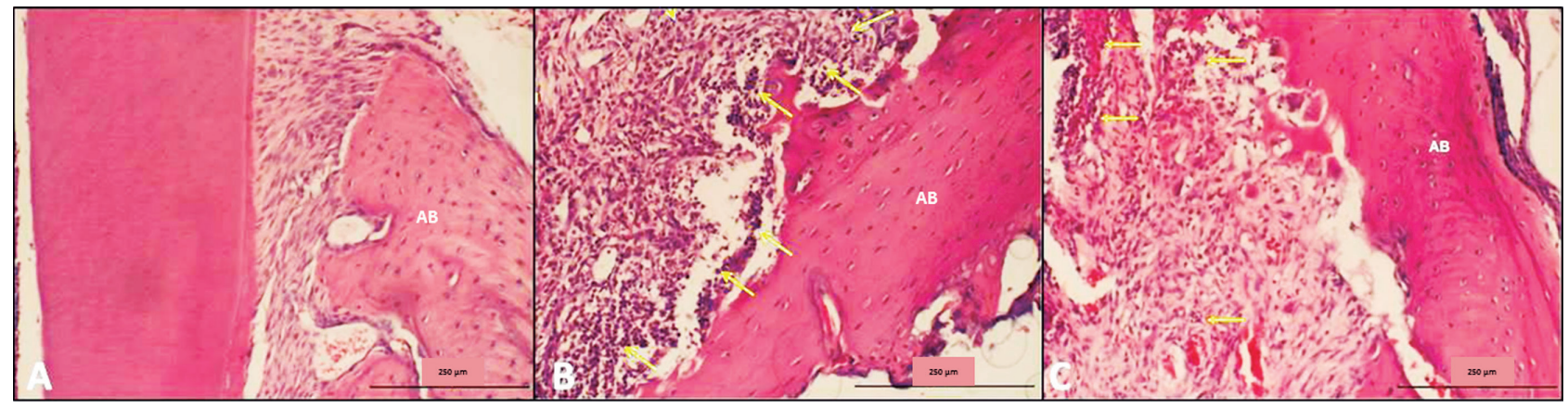

$\mathrm{AB}=$ =alveolar bone; $\mathrm{NL}=$ non-ligated; $\mathrm{LO}=$ ligature only; $\mathrm{S}=$ ligature and treated with sumac

Figure 2- The histological images of each experimental group. A- The histological images of NL group showing normal periodontium (100x magnification); B- The histological images of LO showing intense inflammatory cell infiltrate, dilated blood vessels, and osteoclasts in their Howship's lacunae with multiple resorption foci (100x magnification); C- The histological images of S showing moderate inflammatory cell infiltrate in periodontal ligament and osteoclasts in their Howship's lacunae with multiple resorption foci. Yellow arrows show inflammatory cells (100x magnification) 


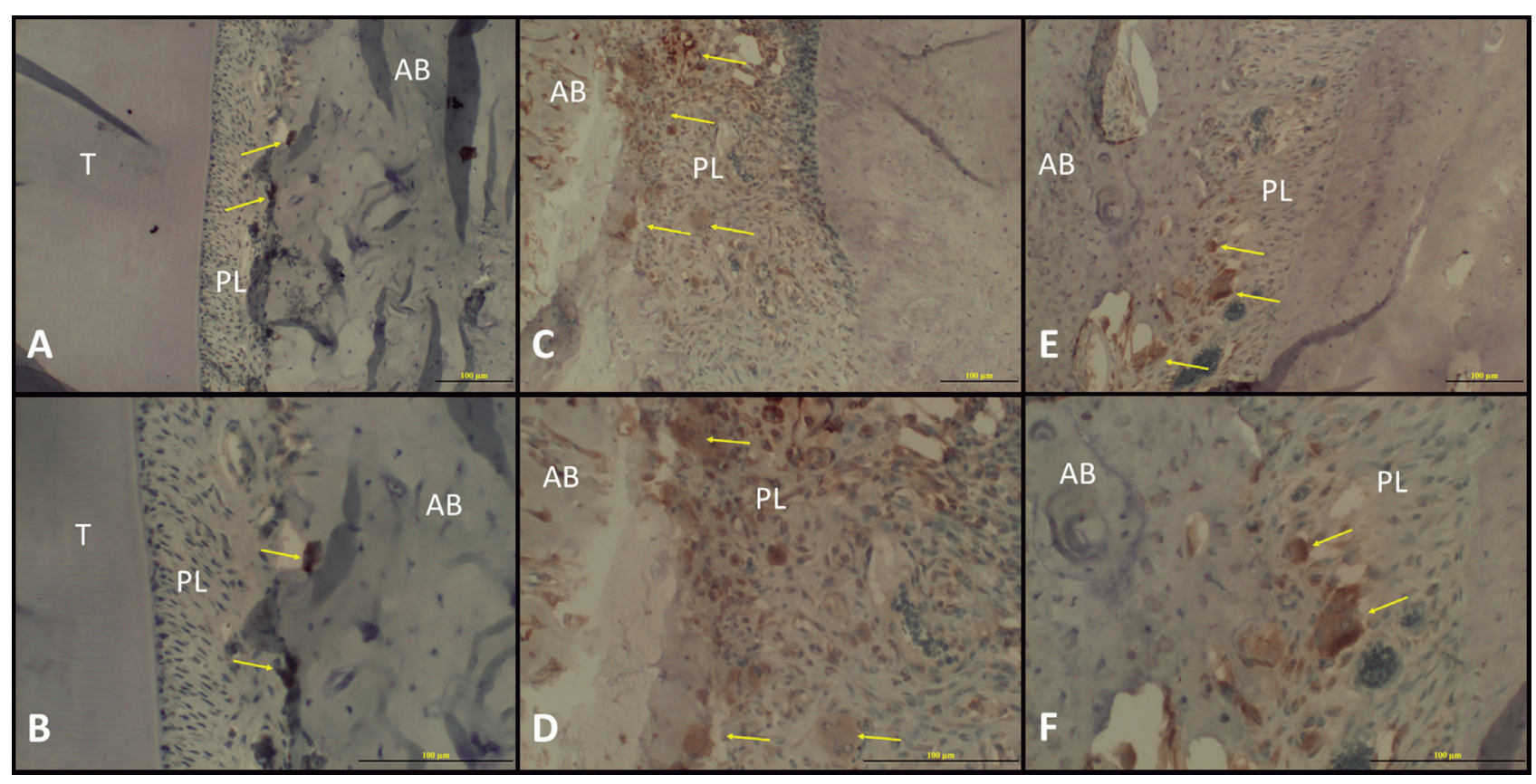

T=tooth; $\mathrm{AB}=$ =alveolar bone; $\mathrm{PL}=$ periodontal ligament; $\mathrm{NL}=$ non-ligated; $\mathrm{LO}=$ ligature only; $\mathrm{S}=$ ligature and treated with sumac

Figure 3- RANKL expressions in NL, LO, and S groups. A- RANKL expressions in NL group at 200x magnification; BRANKL expressions (yellow arrows) in NL group at 400x magnification; C- RANKL expressions (yellow arrows) in LO group at 200x magnification; D- RANKL expressions (yellow arrows) in LO group at 400x magnification; E- RANKL expressions (yellow arrows) in S group at 200x magnification; F- RANKL expressions (yellow arrows) in S group at 400x magnification

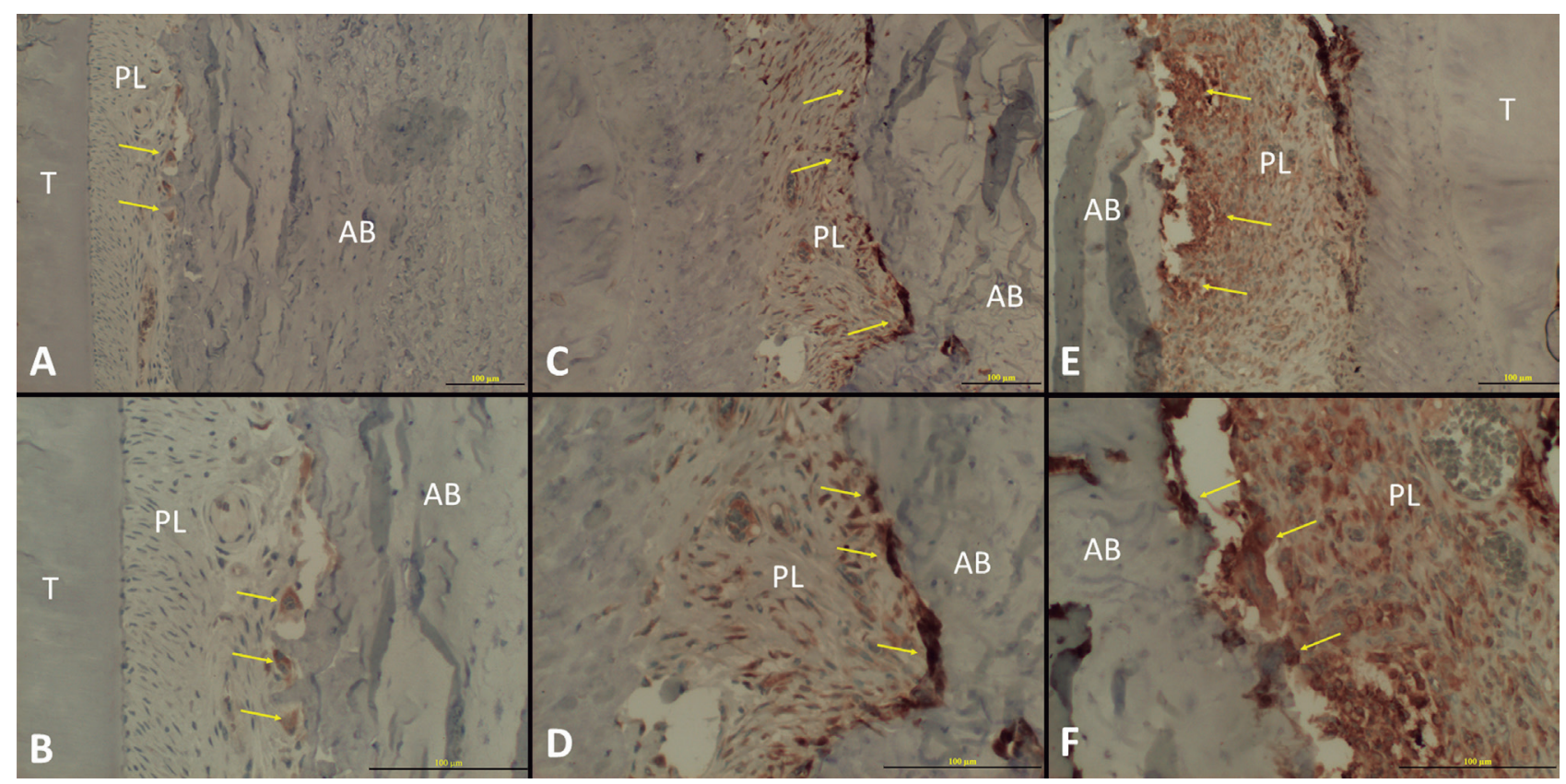

$\mathrm{T}=$ tooth; $\mathrm{AB}=$ alveolar bone; $\mathrm{PL}=$ periodontal ligament; $\mathrm{NL}=$ non-ligated; $\mathrm{LO}=$ =ligature only; $\mathrm{S}=$ =ligature and treated with sumac

Figure 4- Osteoprotegerin (OPG) expressions in NL, LO, and S groups. A- OPG expressions (yellow arrows) in NL group at 200x magnification; B- OPG expressions (yellow arrows) in NL group at 400x magnification; C- OPG expressions (yellow arrows) in LO group at 200x magnification; D- OPG expressions (yellow arrows) in LO group at 400x magnification; E- OPG expressions (yellow arrows) in S group at 200x magnification; F- OPG expressions (yellow arrows) in S group at 400x magnification 
Immunohistochemical findings of RANKL and OPG

Positive RANKL and OPG immunoreactivity was detected in the mononuclear inflammatory cell infiltrate, osteoblasts, osteoclasts, periodontal ligament fibroblasts, and endothelial cells (Figure 3 and Figure 4). Osteoblasts and osteoclasts were dominant stained cells in all specimens. RANKLpositive cells were significantly higher in the LO group than in the $S$ and NL groups $(p<0.05)$. There was also significant difference between $S$ and $\mathrm{NL}$ groups $(p<0.05)$ in terms of RANKL-positive cells (Figure $5 \mathrm{~A}$ ). There were significantly more OPG- positive cells in $\mathrm{S}$ group than in $\mathrm{LO}$ and $\mathrm{NL}$ groups $(p<0.05)$. There was also significant difference between LO and NL groups in terms of OPG-positive cells $(p<0.05)$ (Figure 5B). RANKL/OPG ratio was presented in Figure 5C. RANKL/OPG ratio was lower in $\mathrm{S}$ group compared to NL and LO groups $(\mathrm{p}<0.05)$. There was no significant difference in NL and LO groups $(p>0.05)$.

\section{Serum TAS, TOS and OSI levels}

TAS, TOS and OSI levels were presented for each group in Figure 6.

TAS levels were significantly lower in LO group
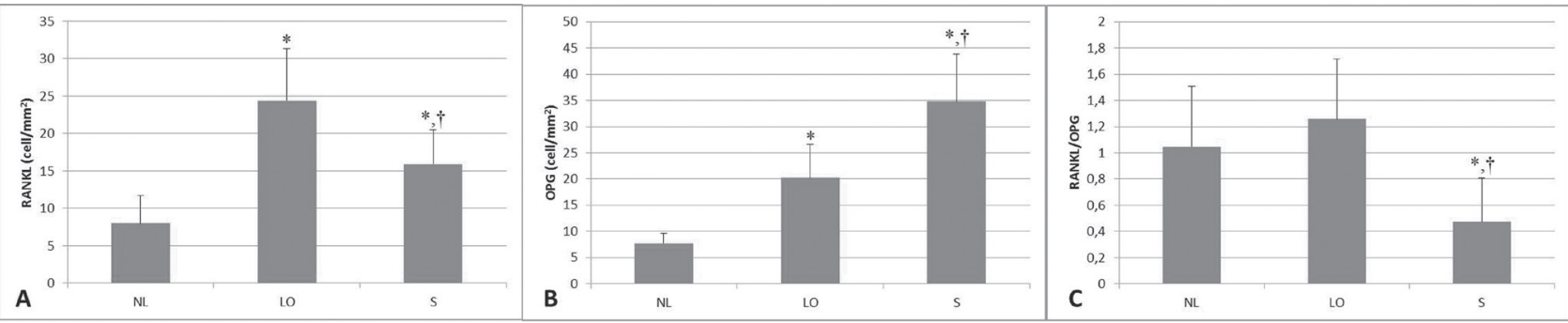

$\mathrm{NL}=$ non-ligated; $\mathrm{LO}=$ ligature only; $\mathrm{OPG}=$ osteoprotegerin; $\mathrm{S}=$ ligature and treated with sumac

Figure 5- A- Mean and standard deviation of RANKL-positive cells in the groups; B- Mean and standard deviation of OPG-positive cells in the groups. *Significant difference compared to NL group. †Significant difference compared to LO; C- RANKL/OPG ratio in the groups
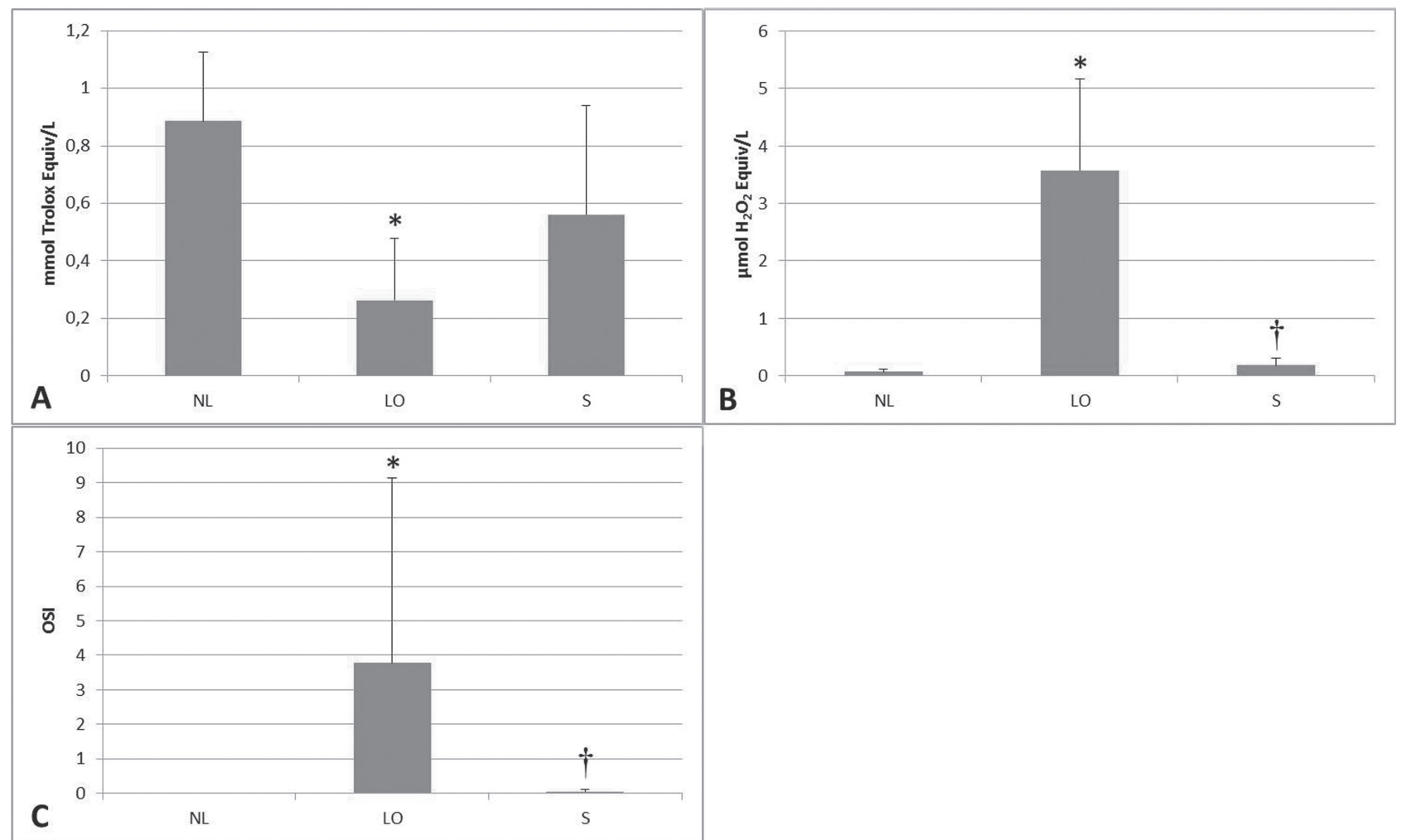

$\mathrm{NL}=$ non-ligated; LO=ligature only; $\mathrm{S}=$ ligature and treated with sumac

Figure 6- A- Total antioxidant status (TAS) in NL, LO, and S groups; B- Total oxidant status (TOS) in NL, LO, and S groups; C- the oxidative stress index (OSI) levels in NL, LO, and S groups. *Significant difference compared to NL group. †Significant difference compared to LO 
compared to NL group $(p<0.05)$. TAS levels were similar in NL and $S$ groups $(p>0.05)$. TAS levels were higher in $\mathrm{S}$ group compared to LO group but it was not statistically significant $(p>0.05)$ (Figure $6 A)$.

TOS levels were significantly higher in LO group compared to NL and S groups $(p<0.05)$. TOS levels were similar in NL and $\mathrm{S}$ groups $(p>0.05)$ (Figure 6B).

OSI levels were significantly higher in LO group compared to NL and $\mathrm{S}$ groups $(\mathrm{p}<0.05)$. OSI levels were similar in NL and $\mathrm{S}$ groups $(p>0.05)$ (Figure $6 C)$.

\section{DISCUSSION}

In the present study, we investigated the effects of sumac extract on alveolar bone loss, RANKL, OPG expressions, TAS, TOS and OSI levels in rat periodontitis model.

ROS have a potential role in tissue destruction associated with inflammatory periodontal diseases. Furthermore, it has been suggested that ROS may act as a physiologic second messenger in RANKL signaling for osteoclastogenesis ${ }^{21}$. It has been demonstrated that ROS triggers RANKL expression through extracellular signal-regulated kinases (ERKs) and protein kinase A-CAMP response element-binding protein (PKA CREB) pathway in mouse osteoblasts and through ERKs and heat shock factor 2 (HSF2) in human MG63 cells ${ }^{4}$. Baek, et al. $^{3}$ (2010) demonstrated that ROS promoted production of RANKL and M-CSF, thus stimulating osteoclastogenesis and osteoclast activity in a primary human bone marrow cell culture. It was suggested that the methanolic extracts of sumac fruits have remarkable antioxidant effect to free radicals and lipid peroxidation in vitro ${ }^{9}$. In another study, it was demonstrated that supplementation of the drinking water with sumac extract $(0.02 \mathrm{~g} / \mathrm{kg}$ per animal) in rats reduced the oxidized DNA bases formation in lung, liver, colon, and lymphocytes; also after $y$-irradiation definite effects were observed $^{11}$. Beneficial effect of sumac on antioxidant status and cholesterol level of adult male rabbits was reported ${ }^{10}$. TOS and OSI are indicators of the degree of oxidative stress ${ }^{15}$. In this study, we found that the mean values of serum TOS and OSI levels were higher in the LO group compared to the NL group. Systemic sumac administration significantly reduced TOS and OSI levels compared to LO group. TAS levels were also higher in $\mathrm{S}$ group compared to LO, but it did not reach the statistically significance. Our data demonstrated that sumac reduced alveolar bone loss and RANKL expression compared to LO group. Considering the relationship between RANKL and ROS, sumac might reduce RANKL expression by reducing oxidative stress because of ROS in our study. In a human study, it was reported that strong negative correlations between OPG and total oxidant status were observed in gingival crevicular fluid and serum samples from periodontitis patients ${ }^{5}$. Elevated OPG level in $\mathrm{S}$ group may be due to reduced total oxidant status in this present study. The sumac extract may also reduce RANKL expression by increasing OPG expression via decreased oxidative stress. It was also demonstrated that RANKL inhibition via systemic delivery of OPG suppresses alveolar bone loss in experimental periodontitis ${ }^{19}$. Considering our data, both RANKL and OPG were increased in LO group. Garlet, et al. ${ }^{19}$ (2004) demonstrated that the intensity of both RANKL and OPG expression was increased in diseased tissues [aggressive (AP) and chronic periodontitis (CP) tissues] compared to healthy tissues ${ }^{17}$. The results of this human study were also in agreement with our findings. It was suggested that RANKL/OPG ratio could be a good indicator of the occurrence of periodontitis ${ }^{7}$. In a recent study, it was demonstrated that RANKL/OPG ratio was significantly higher in active periapical lesions compared to inactive periapical lesions and healthy controls ${ }^{2}$. In that study, it was also demonstrated that higher RANKL/OPG ratio was characterized by the highest TNF- $\alpha$ and RANKL levels among active lesions, and relatively high IL21, IL-17, and IFN-y expression ${ }^{2}$. We observed a significant effect of sumac on RANKL/OPG balance in this study. RANKL/OPG ratio was lower in S group compared to LO group. Significantly decreased RANKL expression and increased OPG expression in $\mathrm{S}$ group compared to LO group may explain this situation.

Although osteoblast numbers were similar in $S$ and LO groups, the lower osteoclast number in S group compared to LO group were observed in our study. It has been shown that oxidative stress decreases the level of bone formation by down-regulating the differentiation and survival of osteoblasts in various in vitro and animal studies ${ }^{3}$. Furthermore, it has been reported that ROS has an important role in activating osteoclasts and thus, promote bone resorption ${ }^{3}$. Decreased osteoclast number in $\mathrm{S}$ group may be explained by inhibitory effect of sumac on oxidative stress based on ROS levels in our study.

The anti-inflammatory effect of sumac extract has been also mentioned in the literature ${ }^{26,29}$. It has been indicated that lyophilized hydroalcoholic crude extract of sumac leaves reduced nitric oxide and prostaglandins $E_{2}$ induced by interleukin- $1 \beta$, which have essential role in inflammation ${ }^{29}$. It was also known that ROS can cause tissue damage by stimulation of pro-inflammatory cytokine release by monocytes and macrophages ${ }^{12}$. According to this finding, sumac extract may show an anti- 
inflammatory effect because of its antioxidant activity. In this present study, inflammatory cell infiltrate was lower in $\mathrm{S}$ group compared to LO group. This observation may be related to antiinflammatory effect of sumac.

The antibacterial effect of sumac has been reported in the literature. Kermanshah, et al. ${ }^{20}$ (2014) demonstrated that Rhus coriaria had antibacterial effects against cariogenic bacteria such as Streptococcus mutans, Lactobacillus rhamnosus, and Actinomyces viscosus. Homer, et al. ${ }^{18}$ (1992) reported that tannic acid, which is present in sumac extract, exerted inhibitory effect on the proteolytic activities of the periodontopathic bacteria Porphyromonas gingivalis, Bacteroides intermedius, and Treponema denticola. In this present study, sumac extract may show an antibacterial effect on these periodontopathogens through tannic acid which exists in its content. This condition may be another explanation why alveolar bone loss is lower in S group compared to LO group in our study.

Sumac has been used mainly in traditional and folk medicine. Therefore, it is difficult to pinpoint the exact recommended standard dose for this herb. Different dosages of sumac extract have been used by researchers. For evaluating antioxidant effects, $200 \mathrm{mg} / \mathrm{kg}$ sumac extract was used orally in the study of Abbass, et al. ${ }^{1}$ (2012). For evaluating antidiabetic effects, 200 and 400 $\mathrm{mg} / \mathrm{kg}$ sumac extract was used orally in another study ${ }^{23}$. Chakraborty, et al. ${ }^{11}$ (2009) reported that the average amount of the sumac extract added to drinking water consumed by the animals per day was $0.02 \mathrm{~g} / \mathrm{kg}$ per animal in their study. We used the same dose (20 mg/kg per day) and the same concentration orogastrically as in their study ${ }^{11}$.

\section{CONCLUSION}

In this present study, administration of ethanol extract of sumac reduced periodontal inflammation, RANKL expression, alveolar bone loss, TOS and OSI levels and increased OPG expression in experimental periodontitis. It was possible to conclude that the ethanol extract of sumac reduced alveolar bone loss by affecting RANKL/OPG balance, TOS and OSI levels in periodontal disease in rats, but further clinical studies are needed.

\section{ACKNOWLEDGEMENTS}

The authors report no conflicts of interest related to this study. No external funding, apart from the support of the authors, was available for this study. Authors are grateful to Bulent Ozkan for his help in statistical analyses and Niyazi Dundar for his help in TAS, TOS and OSI levels analyses.

\section{REFERENCES}

1- Abbass MM, Mahmoud AH, Hussein MM, Gabr SA. Assessment of antioxidant changes of aged rats treated with sumac extract. ] Am Sci. 2012;8:553-8.

2- Araujo-Pires AC, Francisconi CF, Biguetti CC, Cavalla F, Aranha $A M$, Letra $A$, et al. Simultaneous analysis of $T$ helper subsets (Th1, Th2, Th9, Th17, Th22, Tfh, Tr1 and Tregs) markers expression in periapical lesions reveals multiple cytokine clusters accountable for lesions activity and inactivity status. J Appl Oral Sci. 2014;22:336-46.

3- Baek KH, Oh KW, Lee WY, Lee SS, Kim MK, Kwon HS, et al. Association of oxidative stress with postmenopausal osteoporosis and the effects of hydrogen peroxide on osteoclast formation in human bone marrow cell cultures. Calcif Tissue Int. 2010;87:22635 .

4- Bai XC, Lu D, Liu AL, Zhang ZM, Li XM, Zou ZP, et al. Reactive oxygen species stimulates receptor activator of NF-kappaB ligand expression in osteoblast. J Biol Chem. 2005;280:17497-506.

5- Baltacioglu E, Kehribar MA, Yuva P, Alver A, Atagün OS, Karabulut $E$, et al. Total oxidant status and bone resorption biomarkers in serum and gingival crevicular fluid of patients with periodontitis. J Periodontol. 2014;85:317-26.

6- Bartold PM, Cantley MD, Haynes DR. Mechanisms and control of pathologic bone loss in periodontitis. Periodontol 2000. 2010;53:55-69.

7- Belibasakis GN, Bostanci N. The RANKL-OPG system in clinical periodontology. J Clin Periodontol. 2012;39:239-48.

8- Bursal E, Koksal E. Evaluation of reducing power and radical scavenging activities of water and ethanol extracts from sumac (Rhus coriaria L.). Food Res Int. 2011;44:2217-21.

9- Candan F, Sökmen A. Effects of Rhus coriaria L. (Anacardiaceae) on lipid peroxidation and free radical scavenging activity. Phytother Res. 2004;18:84-6.

10- Capcarova M, Slamecka J, Abbas K, Kolesarova A, Kalafova A, Valent $M$, et al. Effects of dietary inclusion of Rhus coriaria on internal milieu of rabbits. J Anim Physiol Anim Nutr (Berl). 2012;96:459-65.

11- Chakraborty A, Ferk F, Simić T, Brantner A, Dusinská M, Kundi $\mathrm{M}$, et al. DNA-protective effects of sumach (Rhus coriaria L.), a common spice: results of human and animal studies. Mutat Res. 2009;661:10-7.

12- Chapple IL. Reactive oxygen species and antioxidants in inflammatory diseases. J Clin Periodontol. 1997;24:287-96.

13- Chapple IL, Matthews JB. The role of reactive oxygen and antioxidant species in periodontal tissue destruction. Periodontol 2000. 2007;43:160-232.

14- Erel O. A novel automated direct measurement method for total antioxidant capacity using a new generation, more stable ABTS radical cation. Clin Biochem. 2004;37:277-85.

15- Erel O. A new automated colorimetric method for measuring total oxidant status. Clin Biochem. 2005;38:1103-111.

16- Ferk F, Chakraborty A, Jäger W, Kundi M, Bichler J, Mišík M, et al. Potent protection of gallic acid against DNA oxidation: results of human and animal experiments. Mutat Res. 2011;715:61-71. 17- Garlet GP, Martins W Jr, Fonseca BA, Ferreira BR, Silva JS. Matrix metalloproteinases, their physiological inhibitors and osteoclast factors are differentially regulated by the cytokine profile in human periodontal disease. J Clin Periodontol. 2004;31:671-9. 18- Homer KA, Manji F, Beighton D. Inhibition of peptidase and glycosidase activities of Porphyromonas gingivalis, Bacteroides intermedius and Treponema denticola by plant extracts. J Clin Periodontol. 1992;19:305-10.

19- Jin Q, Cirelli JA, Park CH, Sugai JV, Taba M Jr, Kostenuik PJ, et al. RANKL inhibition through osteoprotegerin blocks bone loss in experimental periodontitis. J Periodontol. 2007;78:1300-8. 20- Kermanshah H, Kamangar SS, Arami S, Kamalinegad M, Karimi $M$, Mirsalehian A, et al. The effect of hydro alcoholic extract of seven plants on cariogenic bacteria - an in vitro evaluation. Oral Health Dent Manag. 2014;13:395-401. 
21- Lee NK, Choi YG, Baik JY, Han SY, Jeong DW, Bae YS, et al. A crucial role for reactive oxygen species in RANKL-induced osteoclast differentiation. Blood. 2005;106:852-9.

22- Menezes R, Garlet TP, Letra A, Bramante CM, Campanelli AP, Figueira RC, et al. Differential patterns of receptor activator of nuclear factor kappa B ligand/osteoprotegerin expression in human periapical granulomas: possible association with progressive or stable nature of the lesions. J Endod. 2008;34:932-8.

23- Mohammadi S, Montasser Kouhsari S, Monavar Feshani A. Antidiabetic properties of the ethanolic extract of Rhus coriaria fruits in rats. Daru. 2010;18:270-5.

24- Nasar-Abbas SM, Halkman AK. Antimicrobial effect of water extract of sumac (Rhus coriaria L.) on the growth of some food borne bacteria including pathogens. Int J Food Microbiol. 2004;97:63-9.

25- Page RC, Kornman KS. The pathogenesis of human periodontitis: an introduction. Periodontol 2000. 1997;14:9-11.
26- Rayne S, Mazza G. Biological activities of extracts from sumac (Rhus spp.): a review. Plant Foods Hum Nutr. 2007;62:165-75. 27- Sağlam M, Hatipoğlu M, Köseoğlu S, Esen HH, Kelebek S. Boric acid inhibits alveolar bone loss in rats by affecting RANKL and osteoprotegerin expression. J Periodontal Res. 2014;49:472-9. 28- Sculley DV, Langley-Evans SC. Salivary antioxidants and periodontal disease status. Proc Nutr Soc. 2002;61:137-43.

29- Shabbir A. Rhus coriaria Linn, a plant of medicinal, nutritional and industrial importance: a review. J Anim Plant Sci. 2012;22:505-12.

30- Toker H, Ozdemir H, Eren K, Ozer H, Sahin G. N-acetylcysteine, a thiol antioxidant, decreases alveolar bone loss in experimental periodontitis in rats. J Periodontol. 2009;80:672-8. 